\title{
Compositions of Saturn's rings A, B, and C from high resolution near-infrared spectroscopic observations
}

\author{
F. Poulet ${ }^{1}$, D. P. Cruikshank ${ }^{2}$, J. N. Cuzzi ${ }^{2}$, T. L. Roush ${ }^{2}$, and R. G. French ${ }^{3}$ \\ 1 IAS, Bâtiment 121, Université Paris-Sud, 91405 Orsay Cedex, France \\ 2 NASA Ames Research Center, MS 245-3, Moffett Field, CA 94035-1000, USA \\ 3 Astronomy Department, Wellesley College, Wellesley, MA 02481, USA
}

Received 6 May 2003 / Accepted 24 July 2003

\begin{abstract}
We used the NASA IRTF spectrograph SpeX to obtain near-infrared spectra (0.9-5.4 $\mu \mathrm{m})$ of Saturn's rings, achieving spectral resolution $\lambda / \Delta \lambda$ of about 2000. The spatial resolution (about $1 \operatorname{arcsec}$ ) is sufficient to distinguish the three main ring components (A, B and C rings) from one another. These new observations of Saturn's rings are the first to combine an extended spectral range with high spectral resolution and good spatial resolution. We combined these data with recent photometric observations acquired by HST in the $0.3-1.0 \mu \mathrm{m}$ range. The spectra of the A band B rings are dominated by strong features due to crystalline water ice. The shape and the depth of these absorptions differ for each ring, which indicates different water ice grain sizes and abundances. No spectral evidence for volatile ices other than water ice has been detected. Both the lower albedo and the less blue slope in the near-infrared reflectance of the $\mathrm{C}$ ring indicate a concentration of dark material different from that in the A and B rings. The broader triangular Fresnel reflection peak at $3.1 \mu \mathrm{m}$ may support the presence of some amount of amorphous ice. The $\mathrm{C}$ ring spectrum exhibits bands centered at 1.73 and $3.4 \mu \mathrm{m}$ which agree in position quite well with the $\mathrm{C}-\mathrm{H}$ bands. Although the detection is probable, it requires confirmation. With a radiative transfer model, we constrain the grain sizes and the relative abundances of water ice, a dark colorless component (amorphous carbon) to adjust the albedo and a second contaminant to reproduce the reddening in the UV-visible range represented here by organic tholins. The dark component of the $\mathrm{C}$ ring spectrum is included as an intra-mixture only. The cosmogenic implications of the inferred compositions are discussed.
\end{abstract}

Key words. planets: rings - radiative transfer

\section{Introduction}

Saturn's ring particles have been shown to contain water ice (Lebofsky et al. 1970; Clark 1980). The discovery of a reddening between 0.3 and $0.8 \mu \mathrm{m}$ indicated the presence of some additional non-icy material (Lebofsky \& Fegley 1976). Color images of the rings obtained with Voyager imaging (Smith et al. 1981) clearly showed that the $C$ ring and the Cassini division to be less reddish than the $\mathrm{A}$ and $\mathrm{B}$ rings. Recent reinvestigations of Voyager images suggest that the color of rings varied qualitatively on a radial scale of few hundred kilometers (Estrada \& Cuzzi 1996). These broadband photometric measurements were analysed by Cuzzi \& Estrada (1998) with a modified Hapke-van de Hulst model of their construction. An important result was obtained: the color of rings could be best modeled by a small amount of a reddish absorber such as Titan tholin. Microwave and radiometry measurements indicated an icy surface with certainly less than $10 \%$ by mass of non-icy material, but still unidentified (Epstein et al. 1984). A hint of an impurity band near $0.85 \mu \mathrm{m}$ was reported by Clark (1980).

Send offprint requests to: F. Poulet, e-mail: francois.poulet@ias.fr
Recently, the composition of the rings has been studied by modeling a composite spectrum $(0.3-4.0 \mu \mathrm{m})$ of the overall main rings with a Shkuratov-type albedo model by Poulet \& Cuzzi (2002). This study confirmed that the reddish absorber likely is represented by tholins which are molecularly mixed with water ice particles. Three different typical sizes of water ice grain (typically 10, 100 and $1000 \mu \mathrm{m}$ ) are required to reproduce the near-infrared part of the composite spectrum, and a dark material is intimately mixed ("salt-and-pepper" mixture) with water ice grains to lower the albedo. An attempt to reproduce a possible $0.85 \mu \mathrm{m}$ weak absorption with various sets of laboratory data was unsuccessful.

Since the Voyager 2 encounter, only a few ground-based observations have been made. Ring color analysis from Hubble Space Telescope (HST) photometric images revealed a color relation with the small inner satellites (Poulet al. 1999). Cuzzi et al. (2002) studied the color differences at high spatial resolution from new HST images between 0.3 and $1.0 \mu \mathrm{m}$. They suggest the presence of a number of compositionally distinct unidentified materials with different radial distributions.

In this paper, we present the spectral analysis of new near-infrared observations of Saturn's rings, obtained with the 
spectrometer SpeX mounted on the NASA IRTF Telescope, Mauna Kea. These new observations have a spectral resolution much higher than any previous observations. The $0.8-$ $5.5 \mu \mathrm{m}$ spectral range of the instrument covers diagnostic spectral features of a variety of interesting candidate materials: iron-bearing materials at $0.85 \mu \mathrm{m}$, organic materials which could present $\mathrm{C}-\mathrm{H}$ and/or $\mathrm{C}-\mathrm{N}$ stretch features in the 1.7-1.8, 2.2-2.4, 3.2-3.4 and 4.3-4.7 $\mu \mathrm{m}$ regions, $\mathrm{NH}_{3}$ hydrates, which present features near 1.5, 2.2 and $2.4 \mu \mathrm{m}$. Water ice itself also has a strong $3.0 \mu \mathrm{m}$ absorption feature in which very small quantities of dark non-icy material may reveal their presence. Another kind of constituent about which nothing is known is represented by the volatile ices other than water. Because of their proximity to the Sun, the ring particles are unlikely to retain the most volatile ices $\left(\mathrm{CH}_{4}, \mathrm{CO}, \mathrm{N}_{2}\right)$, but the high spectral resolution of these data could reveal absorptions due to $\mathrm{CO}_{2}$ or $\mathrm{CH}_{3} \mathrm{OH}$ ice (Brown 2000). In addition to their spectral resolution, these data have sufficient spatial resolution to distinguish the three main components of the rings ( $\mathrm{A}, \mathrm{B}$ and $\mathrm{C}$ rings), allowing us to test how the composition vary among them.

The phase angle dependence of spectrocopic data has not been well characterized. Cuzzi et al. (2002) and Poulet et al. (2002a) show how the wavelength-dependent reflectivity in the UV-visible range can significantly vary with phase even over 0-6 ${ }^{\circ}$. However, no data exist for longer wavelengths. A strong phase angle dependent spectrum could complicate the interpretation of spectroscopic data. We thus studied the effect of phase angle on the shape of spectra.

The paper is organized as follows. We first present the observations and their reduction. The second part is dedicated to the analysis of Saturn's ring spectra. In particular, modeling of observations is performed with the Shkuratov-type albedo model (Shkuratov et al. 1999; Poulet et al. 2002b) in order to improve our understanding of ring compositional variability in quantitative terms. The consequences of the results on the origin and the evolution of rings are then briefly discussed.

\section{Observations and data reduction}

The observations reported here were made with the spectrograph SpeX of the NASA InfraRed Telescope Facility (IRTF) on Mauna Kea during three nights: 6 October 2000, 7 October 2000 and 8 December 2001. The circumstances of the observations are given in Table 1 . Note that the phase angle $\alpha$ is equal to $4.7^{\circ}$ in October 2000 , and close to opposition $\left(\alpha=0.6^{\circ}\right)$ in December 2001. The October (resp. December) nights had an average seeing of 0.9 arcsec (resp. 1.1 arcsec).

SpeX is a medium-resolution $0.8-5.5 \mu \mathrm{m}$ cryogenic spectrograph, which provides simultaneous wavelength coverage by using prism cross-dispersers at spectral resolutions of $R=$ $\lambda / \Delta \lambda$ approximately 2000 across $0.8-2.5,2.0-4.2$, or $2.4-$ $5.5 \mu \mathrm{m}$ (Rayner et al. 2003). SpeX uses an Aladdin II 1024 $\mathrm{X} 1024 \mathrm{InSb}$ array in its spectrograph and an Aladdin II 512 $\mathrm{X} 512 \mathrm{InSb}$ array in its IR slit-viewer at $0.12 \mathrm{arcsec} / \mathrm{pixel}$. The two cross-dispersed modes were used:

1- The $0.8-2.5 \mu \mathrm{m}$ cross-dispersed mode (mode 1 hereafter); most of this range is covered simultaneously.

2- The 2.4-5.5 $\mu \mathrm{m}$ cross-dispersed mode (mode 2 hereafter);

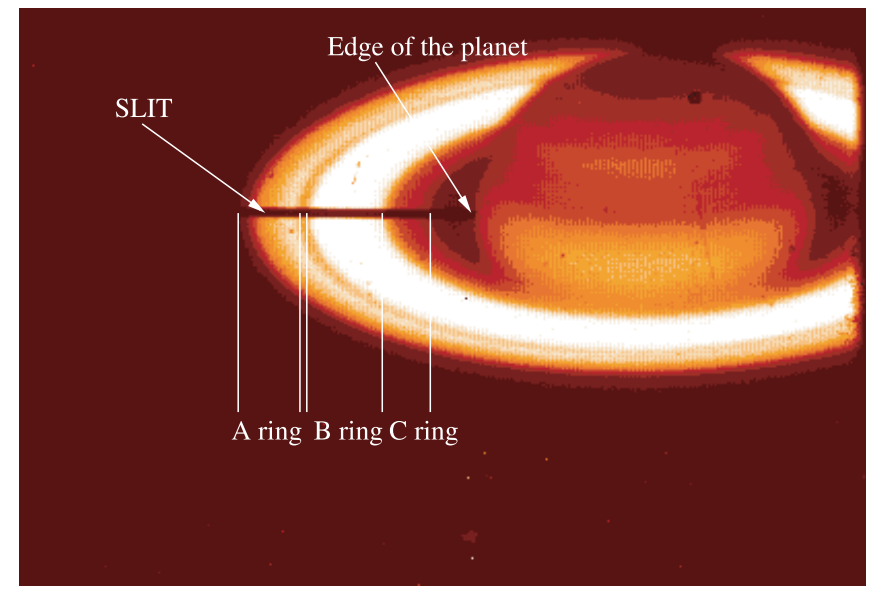

Fig. 1. One IRTF image of Saturn taken in $J$ band on October 6th, 2000 , showing the position of the slit $\left(0.5 \times 15 \operatorname{arcsec}^{2}\right)$ on the west ansa.

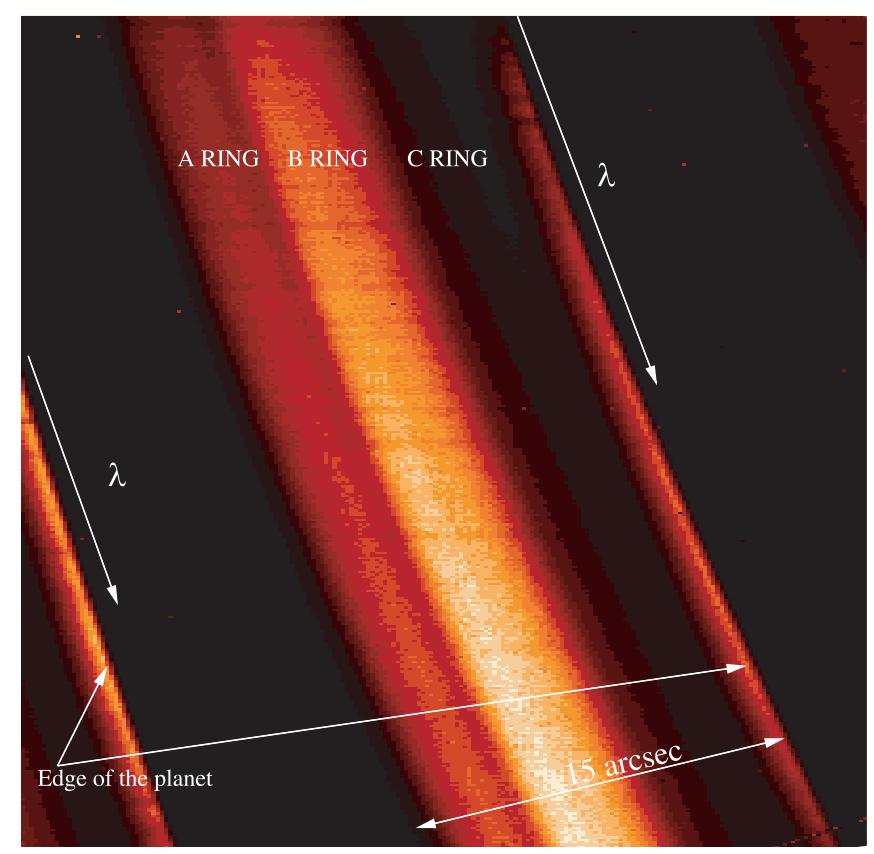

Fig. 2. A part of the mode 1 coverage of the $1 \mu \mathrm{m}$ region obtained with the slit on the rings as shown in Fig. 1. The direction of wavelength dispersion is indicated by $\lambda$. The adjacent partial band is a piece of the next wavelength range, which lies adjacent on the CCD.

with a small rotation of the grating turret, the range $2.0-4.2 \mu \mathrm{m}$ is also covered simultaneously (mode 2 bis hereafter). For these two modes, a slit of $0.5 \times 15 \operatorname{arcsec}^{2}$ was positioned on the rings as shown in Fig. 1.

In October 2000, spectra resulting from six co-added $10 \mathrm{~s} \mathrm{ex-}$ posures for mode 1 and from one exposure of $25 \mathrm{~s}$ for mode 2 bis were measured for each ansa. For mode 2, spectra of three co-added $10 \mathrm{~s}$ exposures were obtained for the east ansa only. Then, we combined different spectra to get a spectrum of $300 \mathrm{~s}$ for mode 1, $300 \mathrm{~s}$ for mode 2 and $350 \mathrm{~s}$ for mode $2 \mathrm{bis}$. For the observations near opposition (December 2001), spectra of $80 \mathrm{~s}$ (ten co-added $8 \mathrm{~s}$ exposures) for mode 1 and $60 \mathrm{~s}$ (three coadded $20 \mathrm{sec}$ exposures) for mode 2 were obtained for each 
Table 1. Observation log.

\begin{tabular}{ccccccc}
\hline \hline Date & Wavelength range $(\mu \mathrm{m})$ & $B^{a}\left({ }^{\circ}\right)$ & $B^{\prime b}\left({ }^{\circ}\right)$ & $\left.\alpha^{c}{ }^{\circ}\right)$ & Air mass & Solar analog star \\
\hline 2000 Oct. 6 & $0.8-2.5,2.0-4.2$ & -24.14 & -23.46 & 4.7 & $1.00-1.13$ & SAO93936 \\
2000 Oct. 7 & $0.8-2.5,2.4-5.5$ & -24.13 & -23.47 & 4.6 & $1.00-1.22$ & SAO93936, SAO94114 \\
2001 Dec. 8 & $0.8-2.5,2.4-5.5$ & -25.85 & -26.104 & 0.6 & $1.00-1.43$ & SAO93936 \\
\hline
\end{tabular}

${ }^{a}$ Ring angle opening as seen from Earth.

${ }^{b}$ Ring angle opening as seen from Sun.

${ }^{c}$ Phase angle.

ansa. Then, we combined different images to get spectra of $400 \mathrm{~s}$ for mode 1 and $600 \mathrm{~s}$ for mode 2 for each ansa. The overall quality of data was improved thanks to the tip-tilt system (an active secondary mirror to reduce the tip-tilt components of seeing and telescope tracking) that was working during the October 2000 observations only.

Because Saturn was too big to be autoguided, guiding was done using manual corrections from the hand-paddle of the telescope control system. The observations were done by switching the slit by 30 arcsec between 2 positions A (planet) and B (sky). Corresponding pairs of images planet/sky were differenced, allowing a first-order subtraction of the sky. The planet/sky images were then flat-fielded, corrected for spatial and spectral distortion using the IDL-based spectral reduction tool called Spextool (Vacca et al. 2003). This program flatfields, extracts and wavelength calibrates our data.

In order to extract the spectra, we averaged the signal along the modes shown in Fig. 2 according to a radial width that depends on the ring:

- width of $1.6 \operatorname{arcsec}(\sim 9500 \mathrm{~km})$ centered in the middle of A ring (122 170-136780 km);

- width of $3.4 \operatorname{arcsec}(\sim 20000 \mathrm{~km})$ centered in the middle of B ring $(92000-117580 \mathrm{~km})$;

- width of $2.0 \operatorname{arcsec}(\sim 12000 \mathrm{~km})$ centered in the middle of C ring (74 510-92000 km).

We calibrated the profiles radially using Saturn's limb and/or the Cassini Division as pointing reference.

Residual background was determined from a linear fit to pixels located outside the $\mathrm{A}$ ring and inside the $\mathrm{C}$ ring, and then subtracted to eliminate the contribution of the planet and the sky. Observations of G-type SAO stars (Table 1) were performed before and after each run of observation of the rings in order to provide atmospheric and photometric calibrations for each mode. The flux- and wavelength- calibrated spectral segments were then merged to provide contiguous spectra between 0.81 and $5.40 \mu \mathrm{m}$.

\section{Analysis of the observations}

Our analysis was performed in two steps. The first step consisted of a description of the spectral characteristics of the three main rings and an assignment of absorption bands. This operation allowed us to select the sets of optical constants for the spectral modeling. The second step consisted of a global modeling of the 0.9-5.4 $\mu \mathrm{m}$ region spectrum, accompanied by the UV/visible spectrophotometric data obtained with HST by Cuzzi et al. (2002) in order to enable a comprehensive analysis of the dark material. Our knowledge of the composition derived from the direct analysis step is thus improved, because quantitative information about the abundance and the grain size of different components can be obtained. Eventually, the implications for the origin and the evolution of rings are discussed.

\subsection{The spectra}

Figure 3 shows our scaled, full-resolution spectra of the A and $\mathrm{B}$ rings at the two phase angles. The signal-to-noise ratio $(S N R)$ of the data taken at $4.7^{\circ}$ was slightly better between 0.9 and $4.2 \mu \mathrm{m}(S N R \sim 100-500)$ and much better beyond $4.5 \mu \mathrm{m}$ than at $0.6^{\circ}$, which provides the first measurement of this part of the spectrum even though the signal is noisy ( $S N R \sim 1-10$ ). The spectra of the two main rings are similar to the spectrum obtained by Clark \& McCord (1980). They resemble those of the higher albedo satellites of Saturn in the $1.0-3.0 \mu \mathrm{m}$ region: they are characterized by a strong blue slope. However, the major water ice features at 1.04, 1.25, 1.50, 1.65, 2.02 and $3.1 \mu \mathrm{m}$ are much more clearly defined than in earlier studies. Moreover, the 1.31 and $1.56 \mu \mathrm{m}$ bands are readily apparent in the $4.7^{\circ}$ spectra. Both the temperature-sensitive band at $1.65 \mu \mathrm{m}$ and the fundamental absorption in the 3.0-3.2 $\mu \mathrm{m}$ region, which appears as a reflection peak because the imaginary index is so large, are in favor of the presence of water ice in its crystalline phase only.

Regarding the presence of ices other than water ice, no spectral evidence for ices of $\mathrm{CH}_{4}$ (major absorption bands at $1.75,1.78,2.20,2.31,2.37 \mu \mathrm{m}), \mathrm{CO}(2.35), \mathrm{N}_{2}(2.14), \mathrm{CH}_{3} \mathrm{OH}$ (2.27), $\mathrm{NH}_{3} \mathrm{OH}(2.20)$ or $\mathrm{CO}_{2}(1.43,2.01,2.07)$ was found. The large noise in the $0.8-0.9 \mu \mathrm{m}$ region, which is just at the edge of our spectral window, prevents the confirmation of a proposed absorption at $0.85 \mu \mathrm{m}$.

While the red slopes of the B and A rings in the UV/visible can vary up to $10 \%$ between $0.6^{\circ}$ and $4.7^{\circ}$ of phase angle (Poulet et al. 2002a), our data show that only slight variations in the spectra are seen in response to differing solar phase angles for $\lambda$ between 0.9 and $2.6 \mu \mathrm{m}$, except between 1.1 and $1.4 \mu \mathrm{m}$ where the $0.6^{\circ}$ data present an unexplained odd peak. For the larger wavelengths, the brightness of the $\mathrm{B}$ ring is noticeably lower at $0.6^{\circ}$ phase angle than $4.7^{\circ}$ phase angle. This effect is especially strong for the A ring, while no data were obtained for the $\mathrm{C}$ ring near the opposition because of the bad SNR. Because we have no instrumental or observational explanations for such large differences, we believe that the $0.6^{\circ}$ phase angle data are 

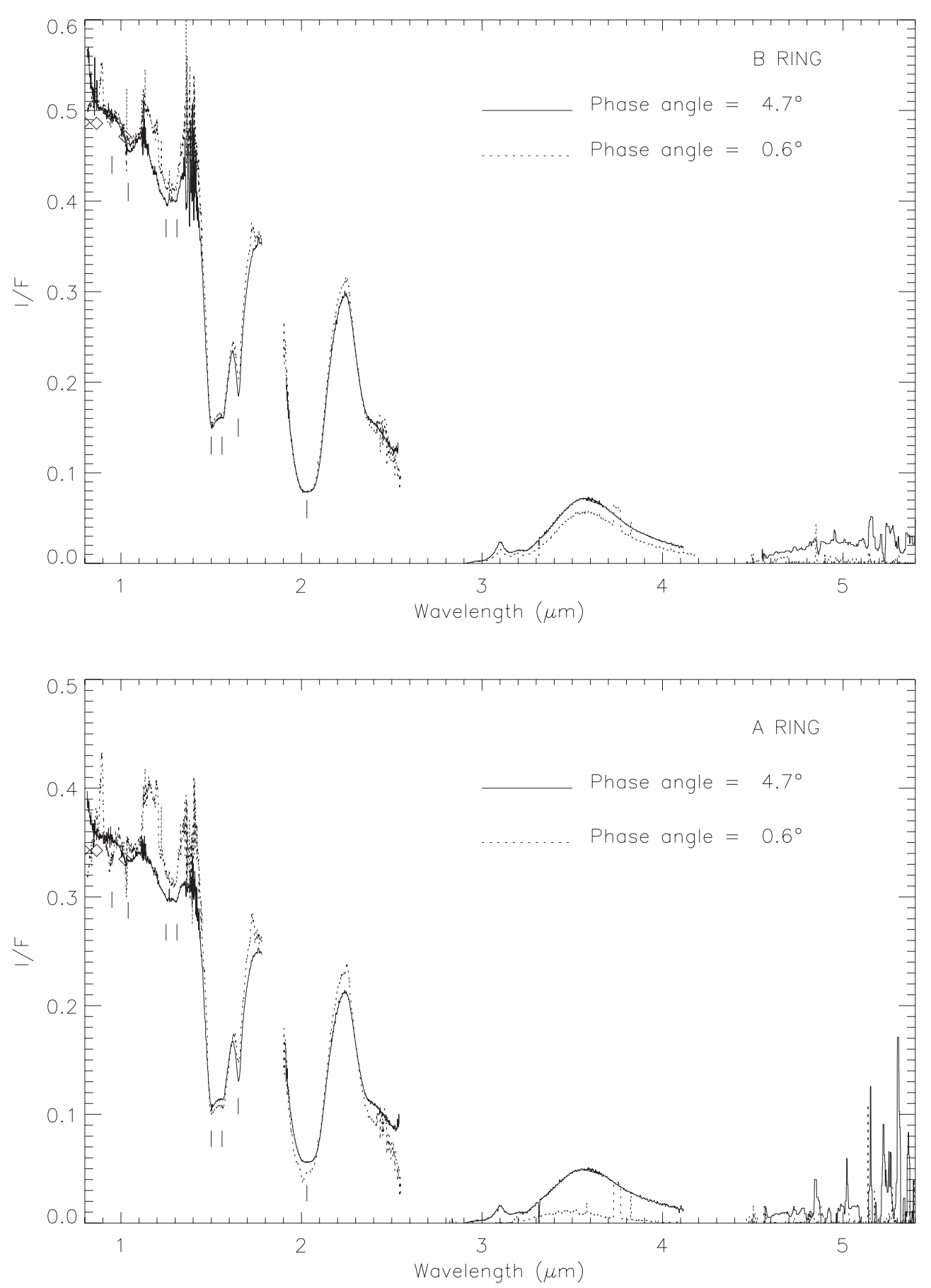

Fig. 3. Spectra of B and A rings at two different phase angles. The water ice absorption bands in the $0.9-2.5 \mu \mathrm{m}$ range are indicated with vertical lines.

suspicious and no reliable conclusion on the phase angle dependence can be therefore made from the present data.

The $\mathrm{A}$ and $\mathrm{B}$ ring spectra are compared using their ratio in Fig. 4. They are very similar, with small differences arising from slightly stronger water ice absorption bands in the $\mathrm{B}$ ring.

The major water ice absorptions are detected in the $\mathrm{C}$ ring spectrum. The $0.95 \mu \mathrm{m}$ band is especially strong. To see it so clearly suggests long path lengths in the ice and is consistent with the strong 1.04 and $1.25 \mu \mathrm{m}$ bands. The overall shape of the spectrum is different from the $\mathrm{A}$ and $\mathrm{B}$ rings (Figs. 4 and 5). In particular, its slope in the near-IR reflectance is less blue.
The blue slope is typical of the pure water ice and depends on the size of surface grains. If dark material is mixed with water ice, the reflectance of the mixture increasingly approaches that of the dark material as its abundance is increased. Thus, both the brightness I/F and the NIR slope of the $\mathrm{C}$ ring compared to $\mathrm{A}$ and $\mathrm{B}$ rings indicate a concentration of dark material different in terms of abundance. On the other hand, the low signal in the $3.0 \mu \mathrm{m}$ region implies that the dark non-icy contaminant is likely incorporated as an intra-mixture (Sect. 3.2) and not as an intimate mixture as in the case of Iapetus (Owen et al. 2001). 

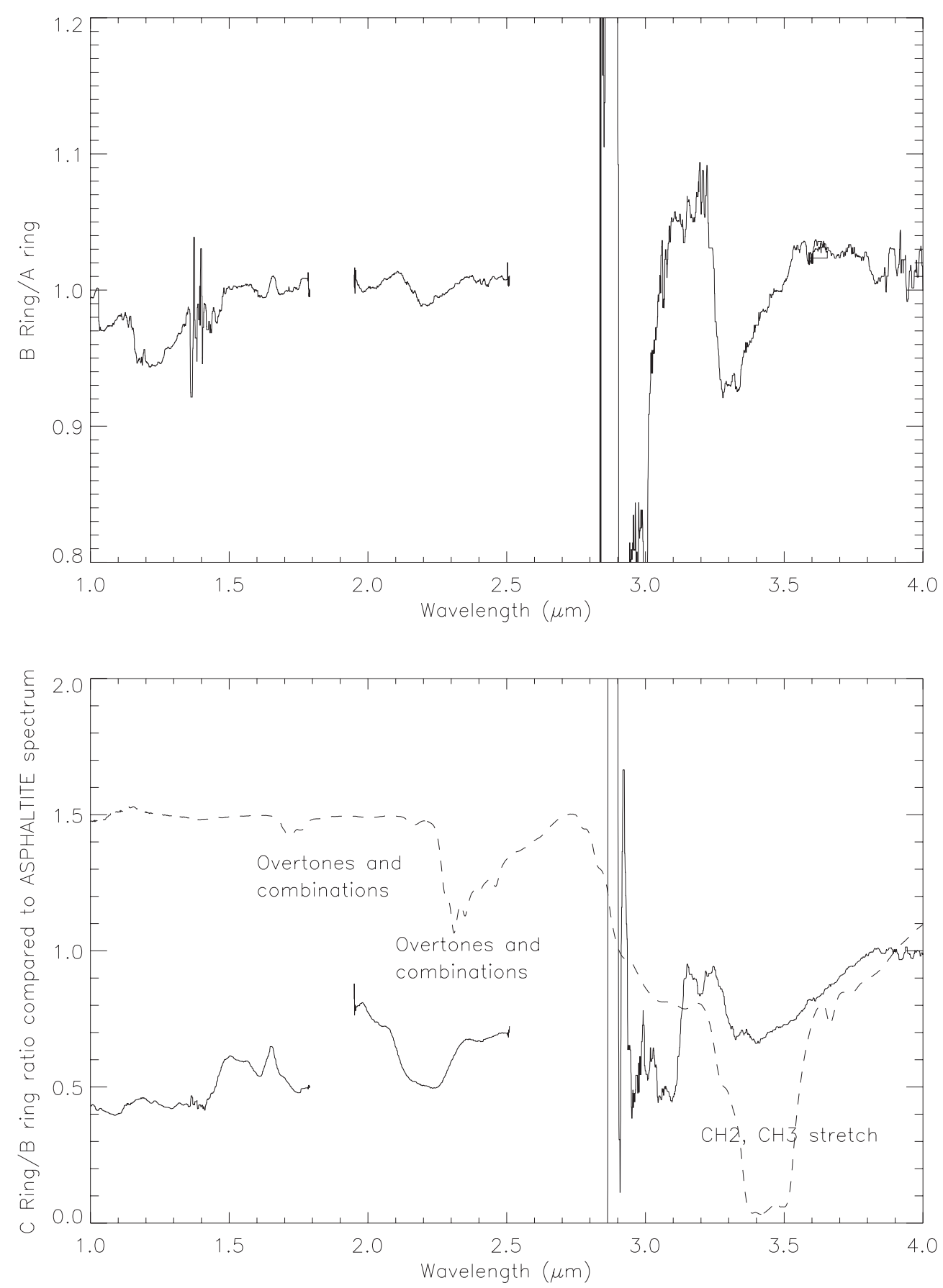

Fig. 4. Upper: Smoothed ratio of spectra of B/A. The ratio is normalized to 1 at $1 \mu \mathrm{m}$. Lower: Smoothed ratio of spectra of C/B compared to a terrestrial and natural organic. The curves are normalized to 1 at $3.9 \mu \mathrm{m}$.

The $1.65 \mu \mathrm{m}$ band clearly implies the presence of crystalline ice. However, its depth in the $\mathrm{C}$ ring is much smaller than in the A and B rings. Using the strong temperature-dependent strength of this feature (Grundy \& Schmitt 1998), we infer an ice physical temperature of about $150 \pm 20 \mathrm{~K}$. This value is much larger than the estimate of $90 \mathrm{~K}$ range from IRIS measurements (Esposito et al. 1984). Although Voyager temperatures were obtained when the rings were almost edge-on to the Sun, this overestimate of the $\mathrm{C}$ ring ice temperature might be also due to the presence of modest quantities of amorphous water ice for which the $1.65 \mu \mathrm{m}$ band disappears. A more convincing argument to probe lattice order is related to the triangular Fresnel reflection peak at $3.1 \mu \mathrm{m}$. As shown in Fig. 6, the peak is broad and low, and its poor resemblance to crystalline ice may also support the presence of amorphous ice. Solid $\mathrm{H}_{2} \mathrm{O}$ at low pressure can exist in two phases: amorphous ice in high and low density configurations, and crystalline cubic and hexagonal phases. The temperature of the phase change from amorphous to crystalline ice is in the range 140-150 K (Jenniskens \& Blake ). Amorphous ice is not commonly found in the Solar System objects even on the very cold surfaces of Charon (Brown \& Calvin 2000) and the uranian satellites 


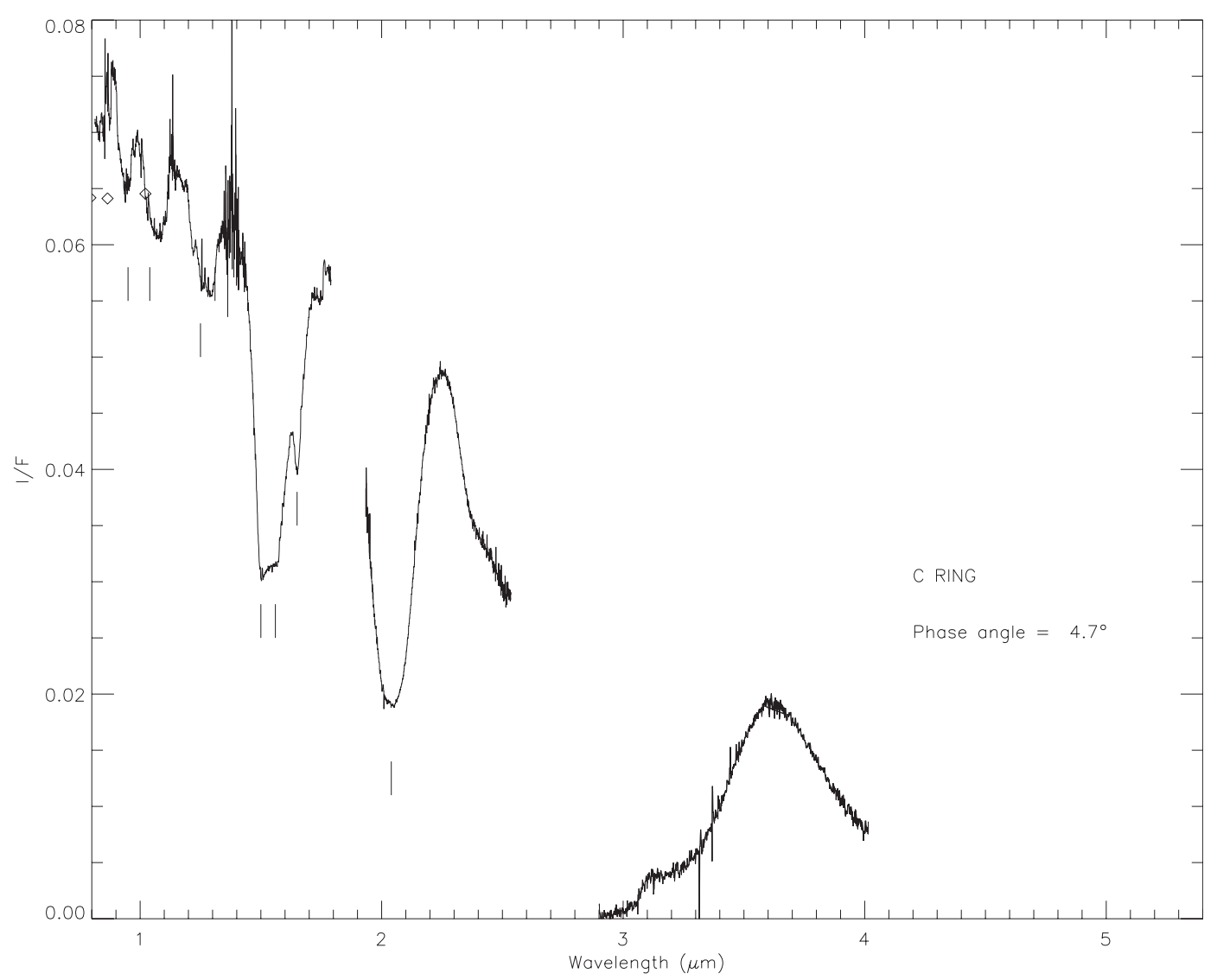

Fig. 5. Spectrum of $\mathrm{C}$ ring obtained at $4.7^{\circ}$ phase angle. The water ice absorption bands in the $0.9-2.5 \mu \mathrm{m}$ range are indicated with vertical lines.

(Roush et al. 1998; Grundy et al. 1999) where it could be stable.

The $\mathrm{C}$ ring spectrum exhibits one band centered at $1.73 \mu \mathrm{m}$. It is likely not a spectral artifact because it does not appear in B and A ring spectra. We compared the spectrum with laboratory transmission spectra of major pure ices $\left(\mathrm{CH}_{4}, \mathrm{CO}, \mathrm{N}_{2}, \mathrm{CH}_{3} \mathrm{OH}\right.$ or $\left.\mathrm{CO}_{2}\right)$. This comparison indicates that this band could be assigned to a band of $\mathrm{CH}_{4}$ ice $\left(v_{2}+v_{3}+v_{4}\right)$ only. However, pure $\mathrm{CH}_{4}$ ice has several other strong bands in the $K$ filter region (at 2.20, 2.31, $2.37 \mu \mathrm{m}$ ) which are not detected. Other potential candidates could be minerals (silicates, salts, and carbonates). We have investigated the mineral hypothesis using the USGS data base (Clark et al. 1993), but none of the main known minerals possess this feature at $1.73 \mu \mathrm{m}$ without other strong features. For salts and carbonate materials, the strong features are in the $3-5 \mu \mathrm{m}$ range.

The $\mathrm{B}$ and $\mathrm{C}$ ring spectra are compared using their ratio in Fig. 4. Much of the structure is due to the greater water ice band strengths in the $\mathrm{B}$ ring compared to the $\mathrm{C}$ ring. However, there are two regions at 1.73 and $3.4 \mu \mathrm{m}$ where the differences cannot be explained by the water ice bands. These bands agree in position quite well with the aliphatic $\mathrm{C}-\mathrm{H}$ streching and bending bands of the spectrum of asphaltite, a natural solid oil bitumen (Moroz et al. 1998). The absorption at 2.3-2.5 $\mu \mathrm{m}$ is not clearly visible in the $\mathrm{C} / \mathrm{B}$ ratio, in part because of the interference of the water ice band stronger in B than in C. This implies that the
$\mathrm{C}-\mathrm{H}$ may be present in the $\mathrm{C}$ ring only, but it needs confirmation.

\subsection{The model}

We use the model recently developed by Shkuratov et al. (1999) to calculate the albedo of a ring particle. This model, based on the geometrical optics approximation as in the more familiar Hapke (1981) model, provides the spectral albedo of powdered surfaces. The degree of physical realism has been studied in Poulet et al. (2002b). In addition to common intimate ("saltand-pepper") mixture, an interesting type of mixture can be formulated: some individual large particles (water ice in the case of ring particles) must themselves contain a mixture of different materials visualized as small inclusions $(\ll \lambda)$ in the bulk. This mixture belongs to the type previously referred to as "intra" or "molecular" mixing of constituents (Cuzzi \& Estrada 1998). The Shkuratov model was successfully used to reproduce a composite spectrum of Saturn's rings (Poulet \& Cuzzi 2002), and more details on the model and our representation of a particle's surface can be found in that paper.

\subsection{The choice of optical constants}

We combined our spectra with UV/visible spectrophotometric data obtained with the HST by Cuzzi et al. (2002) in order to 

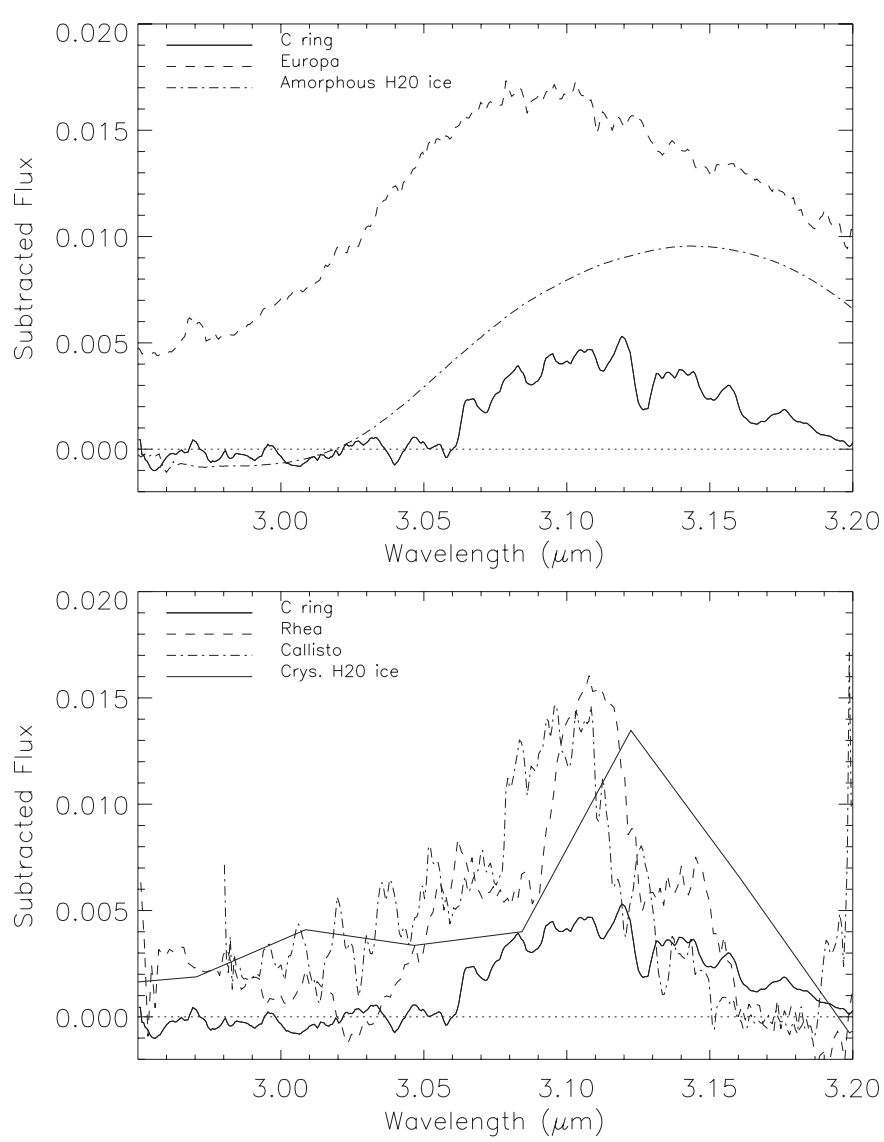

Fig. 6. Comparison of the 2.95-3.2 $\mu \mathrm{m}$ spectra of the $\mathrm{C}$ ring (solid) and several icy satellites with amorphous water ice (upper) and crystalline water ice (lower). A continuum determined by fitting a least squares polynomial curve $2.85-3.3$ points at the ends of the spectral range is subtracted. The spectra shown include calculated spectrum of amorphous frost, laboratory reflectance of crystalline water ice (Roush et al. 1990), spectra of Europa (McCord et al. 1999), and Callisto, Rhea (Dalle Ore 2002, personal communication).

get composite spectra of the $\mathrm{B}$ and A rings from 0.25 to $5.4 \mu \mathrm{m}$ (Figs. 7 and 8 ) and the $\mathrm{C}$ ring from 0.25 to $4.0 \mu \mathrm{m}$ (Fig. 9).

For each ring, the mixture has to satisfy the following constraints:

1. The shape and depth of the various water ice absorptions. The spectroscopic signature of water ice is a diagnostic of the size of grains covering the ring particles (frost). Thus, different grain sizes will be considered. The values of optical constants for crystalline $\mathrm{H}_{2} \mathrm{O}$ ice are taken from Grundy $\&$ Schmitt (1998) between 1.0 and $2.7 \mu \mathrm{m}(T=80 \mathrm{~K})$ and Bertie et al. (1993) beyond $2.7 \mu \mathrm{m}(T=100 \mathrm{~K})$. For the $\mathrm{C}$ ring, amorphous water is also investigated. The optical constants are from Hudgins et al. (1993) above $1.0 \mu \mathrm{m}$. We adopted the values from Warren (1984) between 0.3 and $1.0 \mu \mathrm{m}$ for which the state of the water ice is polycrystalline hexagonal near the melting point. Note that the difference between crystalline and amorphous ices is expected to be small in this wavelength range because the optical constants are so small that it is unlikely to play a role.
2. The red slope from 0.3 to $0.7 \mu \mathrm{m}$. In our previous modeling (Poulet et al. 2002b), we showed that only complex carbon compounds represented by tholins can reproduce the reddening. Therefore, several kinds of tholins are used here: Triton tholin (McDonald et al. 1994), Titan tholin (Khare et al. 1984), two kinds of ice tholins (Khare et al. 1993; Khare, unpublished data).

3. The value of $I / F$, which is not the particle surface reflectance, includes effects of the opacity of rings defined by the standard expression in the single scattering limit (Cuzzi et al. 1984): $\frac{\sin B^{\prime}}{\sin B+\sin B^{\prime}}\left\{1-\exp \left[-\tau\left(\frac{1}{\sin B}+\frac{1}{\sin B^{\prime}}\right)\right]\right\}$. The brightness of the $C$ ring, mid-A ring and inner $B$ ring is fairly well represented by this model, while the outer $\mathrm{B}$ ring shows considerable deviation from this behavior (Cuzzi et al. 2002). This means that the B ring particle composition is less well constrained by the present data than the $\mathrm{A}$ and $\mathrm{C}$ ring particles. Spectrally neutral low-albedo amorphous carbon (Rouleau \& Martin 1991) is included in the scattering calculations mainly to lower the average spectral reflectance and to reduce the contrast in the water ice absorptions, without altering the spectral shape overall.

\subsection{Results}

We simultaneously fit the data for all wavelengths using the IDL simplex minimization algorithm. For the A and B rings, the best-fitting model consists of a salt-and-pepper mixture of four components (Figs. 7 and 8): water ice grains of three different sizes (typically 10, 100 and $1000 \mu \mathrm{m}$, probably merely indicating a grain size distribution) and amorphous carbon grains (Tables 2 and 3). In order to reproduce the red slope, the water ice grains have to be contaminated by a reddish tholin compound. As expected from previous albedo measurements (Doyle et al. 1989; Dones et al. 1992), the amount of dark matter (carbon) is larger for the A ring $(8 \% \mathrm{vol})$ than for the $\mathrm{B}$ ring ( $2 \%$ vol). From a spectrum integrated over the $B$ ring, the Cassini division and a part of A ring, Poulet \& Cuzzi (2002) found a relative abundance of $7 \%$. Note that we cannot constrain the diameter $l$ of carbon particles, because no absorption band exists. A lower limit can be given only as explained in Poulet \& Cuzzi. (2002). We also tried to incorporate the amorphous carbon as both an intramixture and an intimate mixture, but the fitting procedure converged to a minimal solution where no intramixed amorphous carbon is present.

For the $\mathrm{C}$ ring, the best-fitting model consists of a salt-andpepper mixture of crystalline water ice grains of three different sizes intra-mixed with both ice tholin and amorphous carbon inclusions (red line in Fig. 9, Table 4). If the amorphous carbon is intimately mixed as found for the A and B rings, we fail to reproduce the $C$ ring spectrum (green line in Fig. 9): the model is too bright in general and the bands are too shallow. In other words, the carbon, if intimately mixed, seems to provide too large a reflectivity from front-surface grain reflection in the deep water absorptions. By contrast, in the case of amorphous carbon intramixtured, the water ice component can be on the top of amorphous carbon making the deep bands dark enough. We also tested the presence of a small amount of 


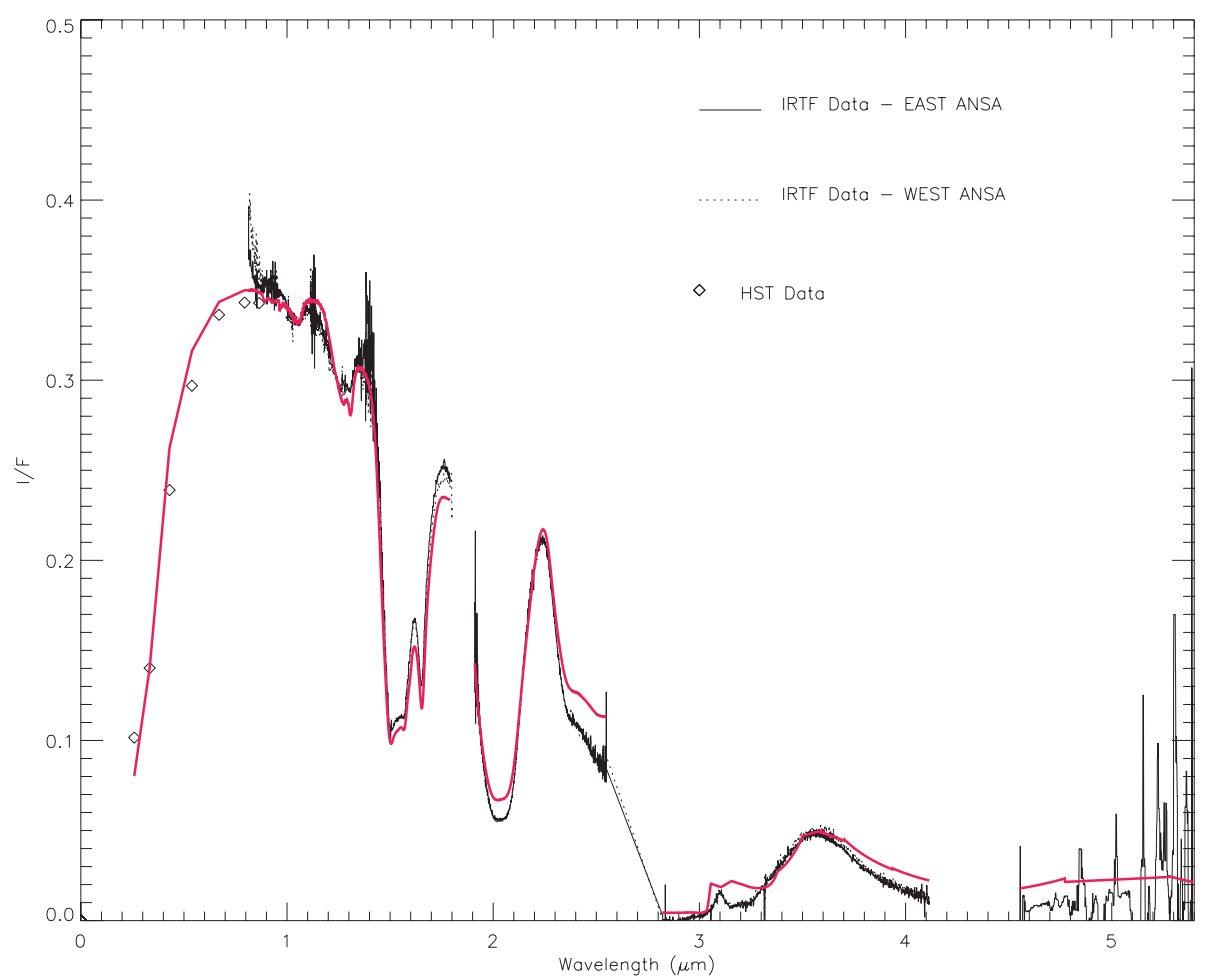

Fig. 7. Spectra of the B ring taken at $4.7^{\circ}$ phase angle compared with its model (red curve). No significant difference between East and West ansa is observed. See Table 2 for a description of the model.

Table 2. B ring: Relative abundances and grain sizes.

\begin{tabular}{ccccc}
\hline \hline Component & Water Ice & Water ice & Water Ice & Amorphous Carbon \\
\hline Abundance $($ vol \%) & $57^{*}$ & $29.7^{*}$ & $11^{*}$ & 2.3 \\
Grain size $(\mu \mathrm{m})$ & 10 & 130 & 1300 & 10 \\
\hline
\end{tabular}

* Contaminated by $0.74 \%$ of small ice tholin inclusions.

Table 3. A ring: Relative abundances and grain sizes.

\begin{tabular}{ccccc}
\hline \hline Component & Water Ice & Water ice & Water Ice & Amorphous Carbon \\
\hline Abundance $($ vol \%) & $46^{*}$ & $31^{*}$ & $15^{*}$ & 8 \\
Grain size $(\mu \mathrm{m})$ & 13 & 130 & 1400 & 10 \\
\hline
\end{tabular}

* Contaminated by $0.53 \%$ of ice tholin inclusions.

Table 4. C ring: Relative abundances and grain sizes (red model in Fig.9).

\begin{tabular}{cccc}
\hline \hline Component & Water Ice & Water ice & Water Ice \\
\hline Abundance $($ vol \%) & $57^{*}$ & $15^{* *}$ & $28^{* *}$ \\
Grain size $(\mu \mathrm{m})$ & 1100 & 7500 & 30 \\
\hline
\end{tabular}

* Contaminated by $0.07 \%$ of amorphous carbon inclusions and $0.41 \%$ of ice tholin inclusions.

** Contaminated by $0.41 \%$ of ice tholin inclusions.

amorphous ice by incorporating a grain made of dirty amorphous ice. However, this worsens the fit to the positions of the water ice absorption bands (blue line in Fig. 9).

\subsection{Discussion}

In order to better identify the discrepancies between the model and the data, we divide the data by the best fit model (Figs. 10 and 11).

There is a significant discrepancy in the UV range (F255W and F336W HST filters). This can be interpreted in two ways: 1- the specific tholins we adopted absorb too strongly in UV, or 2- the rings being a strong red source, the steeply changing intensity across the filter band pass could move the band center to a longer wavelength relative to the band center theoretically defined for a white light source. We reiterate that our fitting procedure consisted of finding the best fit of the overall spectrum. Specifically, the amount of tholin has been derived from the best fit of the reddening from 0.25 to $0.8 \mu \mathrm{m}$. Matching a slope 


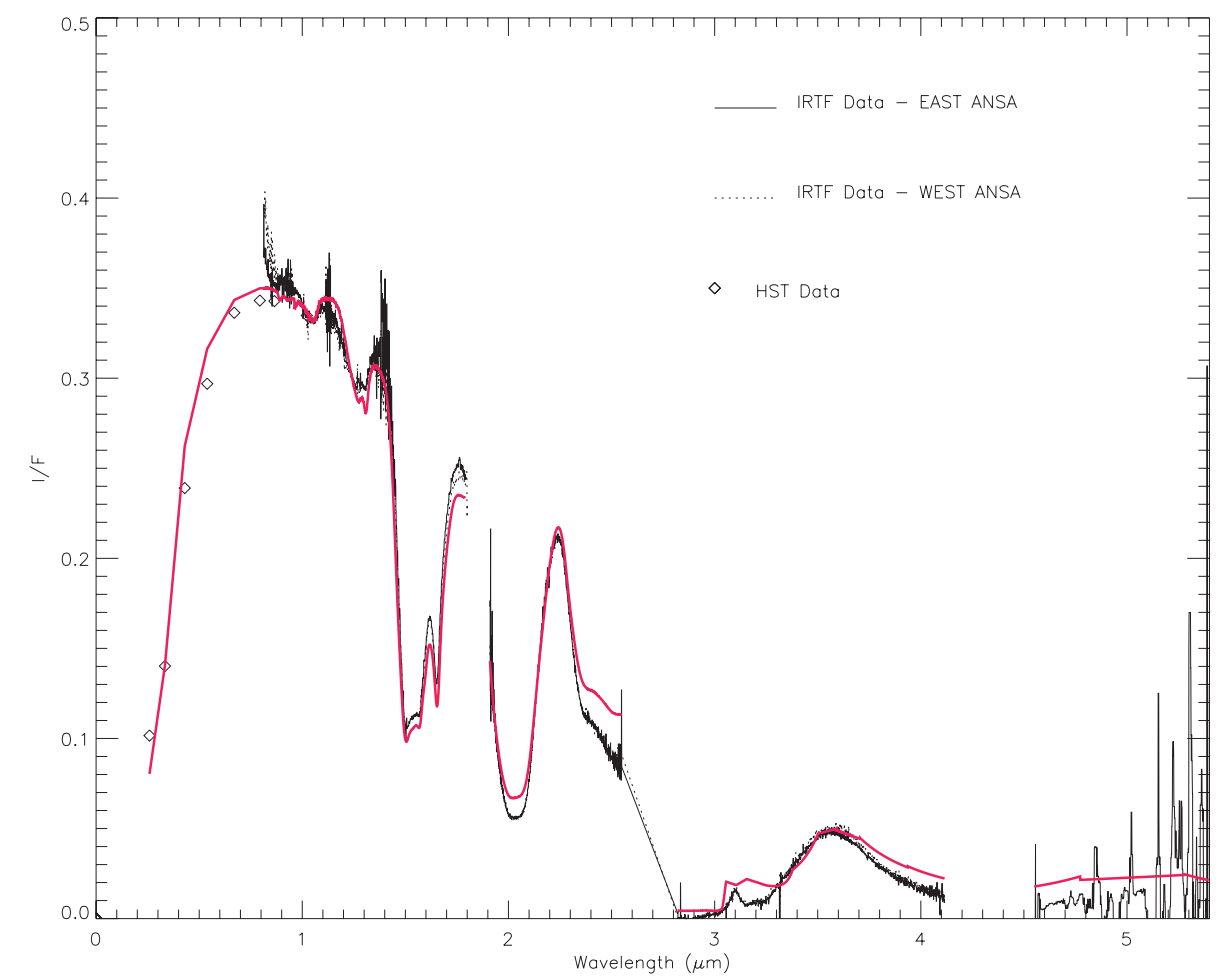

Fig. 8. Spectrum of the A ring taken at $4.7^{\circ}$ phase angle compared with its model (red curve). No significant difference between East and West ansa is observed. See Table 3 for a description of the model.

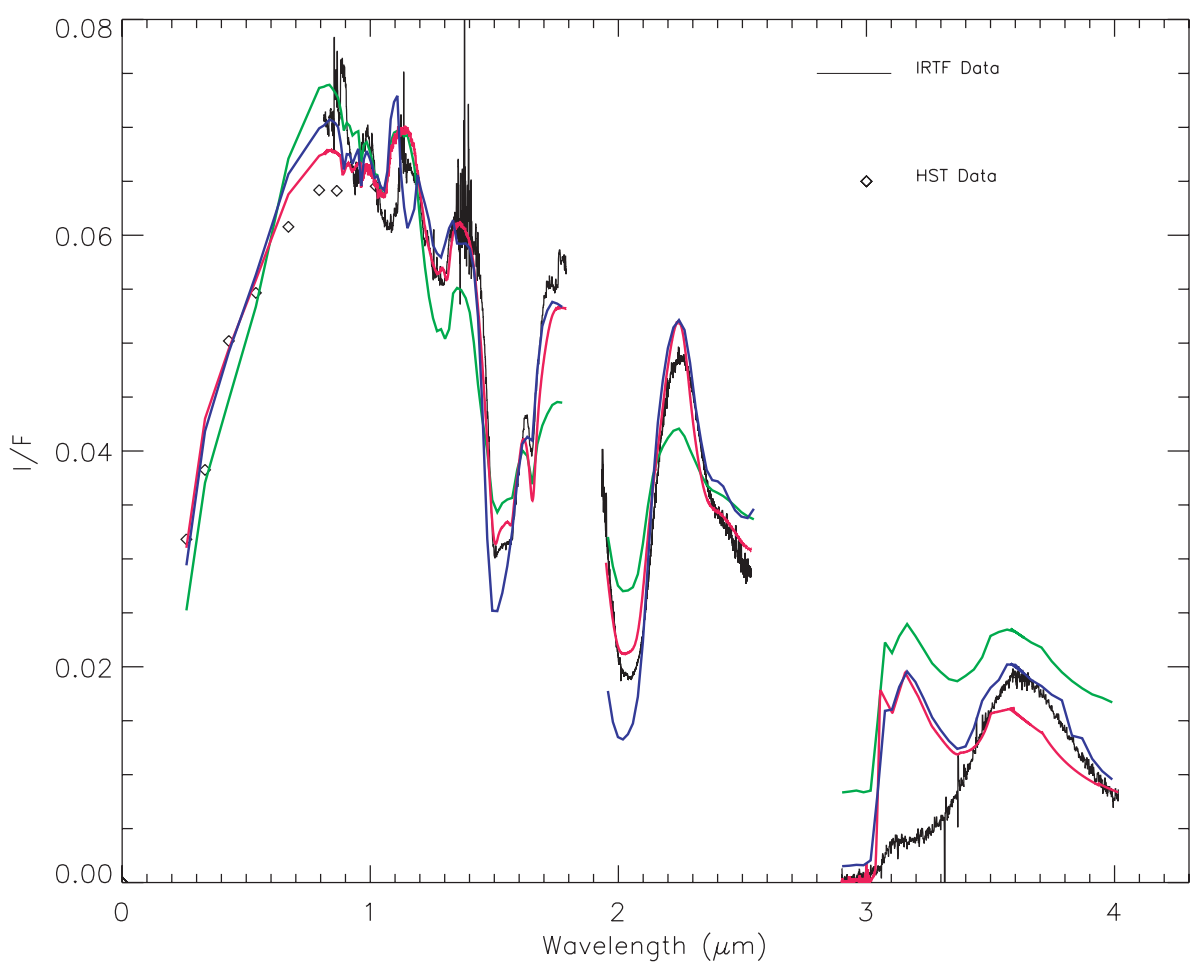

Fig. 9. Spectrum of the $\mathrm{C}$ ring compared with three models (red, green and blue curves). Red: best model; blue: same as red except for one water ice grain made of amorphous water ice; green: Same kind of mixture than that used for the spectra of the B and A rings.

is not a satisfying approach for proving a surface composition. It is suited for proving that something is not present, but not so good for establishing what is present. However, the tholins provide a far better fit than any other known materials (Poulet \& Cuzzi 2002), and do not introduce or interfere with spectral features at other wavelengths. We also note that the red reflectance slope in the same spectral region has been taken as evidence for the presence of organic solid material in the surface materials of other Solar System bodies, notably the Centaur object 5145 Pholus (Cruikshank et al. 1998; Poulet et al. 2002b), 

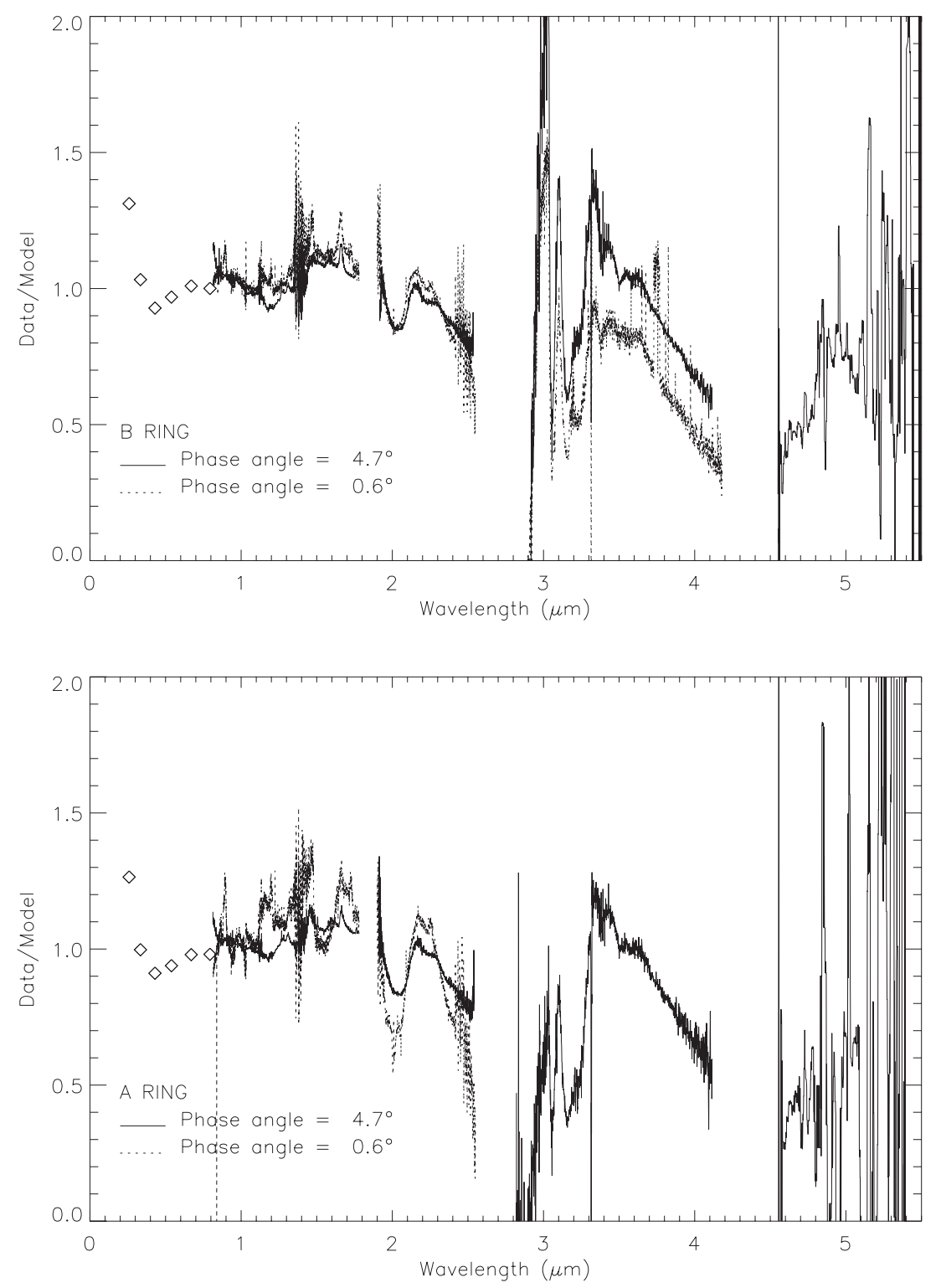

Fig. 10. Data/model ratio for B (upper) and A rings (lower).

because minerals and ices alone do not provide an appropriate absorption in the violet and ultraviolet regions.

Between 1 and $2.5 \mu \mathrm{m}$, the fits agree reasonably well, even if some variations of ratios are due to differences in water ice band shapes (2.0 $\mu \mathrm{m}$ for example). A significant mismatch is observed at wavelengths longer than $2.2 \mu \mathrm{m}$ in the case of $\mathrm{A}$ and $\mathrm{B}$ rings. Water ice alone does not appear to account for the spectra. Some other component is needed to achieve the blue slope. This problem has been already noted in the attempts of modeling of the spectra of the Saturn's bright satellites (Benedix et al. 1998; Dalle Ore et al. 1999; Cruikshank et al. 1999) and Charon (Buie \& Grundy 2000). The possible but unidentified constituent seems to be similar to what is seen on other icy bodies of the outer Solar System.

Obviously, the wavelength region longer than $2.8 \mu \mathrm{m}$ is less well reproduced by the models than the shorter wavelength region. This spectral region has not been a subject of extensive analysis for Solar System objects because of the lack of data with sufficient SNR. Moreover, modeling analysis is not easy because of the combination of the lack of accurate optical constants and the strong dependence on grain size. In spite of these limitations, we attempt to explain the anomalous spectral features below.

A major discrepancy occurs in the $3.0-3.2 \mu \mathrm{m}$ where the water ice has very large imaginary refractive index. The peak comes from the Fresnel reflection off the facets of water grains in the surface, and is thus from zero depth. The saturnian satellites for which data exist in this region, also present this peak (Fig. 6). In any case, the Shkuratov model fails to reproduce the shape of the absorption peak. The presence of frost made of very small grains $(\leq \lambda)$ might reproduce this peak (Hansen, personal communication). However, we recall that the Shkuratov theory, which is based on geometrical optics, is not suitable to simulate the contribution of such a fine frost.

Beyond $3.2 \mu \mathrm{m}$, the shape of the bump strongly depends on water ice grain size and possible presence of unidentified 


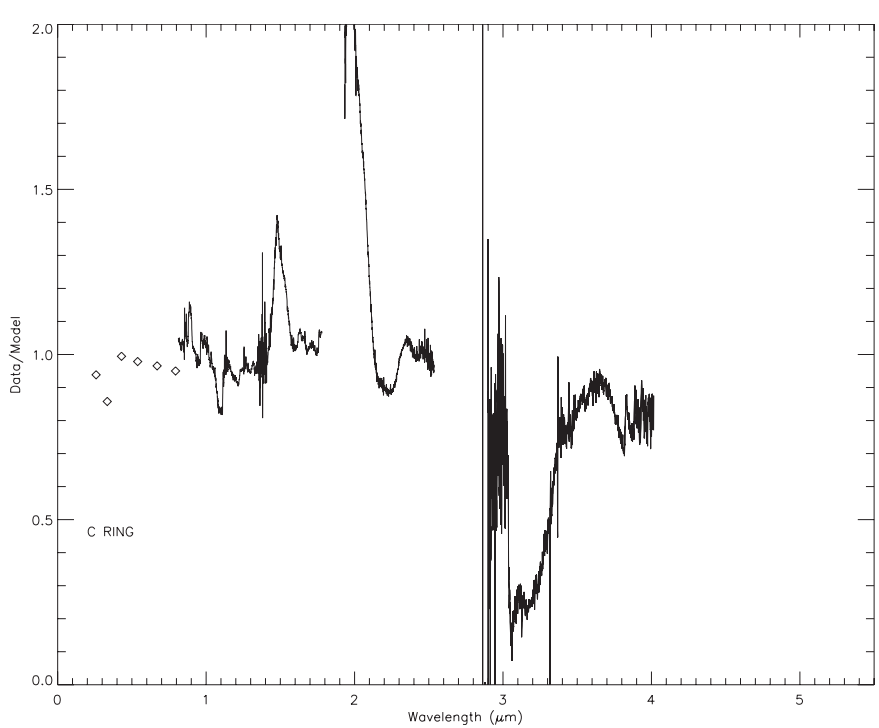

Fig. 11. Data/model ratio for $\mathrm{C}$ ring.

non-icy impurities. Again, the rise to the peak at $3.6 \mu \mathrm{m}$ is higher than the model, indicating further that very fine icy grains and/or some impurities might exist. The entire region beyond $3.6 \mu \mathrm{m}$ is bad because the model overestimates the signal. The presence of the non-icy components used in our modellings is not responsible for the greater signal. Larger grains have a lower albedo beyond $3.6 \mu \mathrm{m}$ but larger grains are ruled out by the shapes of the $\mathrm{H}_{2} \mathrm{O}$ absorption bands at 1.6 and $2.0 \mu \mathrm{m}$. However, it seems quite possible that a component of the absorber may simply be $\mathrm{H}_{2} \mathrm{O}$ with absorptions distorted by close association of other elements or ions. The precise details of the bandshapes depend on the composition, temperature, thermal history, and equilibria established between radiation, erosional and photochemical processes. The optical constants of ring water ice will therfore surely differ from those of laboratory water ice if their conditions of formation or evolution were different.

\section{Conclusion and implications for the origin and evolution of the rings}

These new observations of Saturn's rings are the first to combine an extended spectral range, high spectral resolution, and spatial resolution. The analysis presented here yields new information on the composition of the surfaces of the ring particles in $\mathrm{A}, \mathrm{B}$ and $\mathrm{C}$ rings separately:

- No spectral evidence for ices other than water ice was found.

- The ring particle surface (frost) is made of dirty water ice grains of different sizes (typically 10, 100 and $1000 \mu \mathrm{m}$ ) contaminated by red organic material represented here by tholins. Cuzzi \& Estrada (1998) first demonstrated that organic-bearing material was likely part of the composition of Saturn's rings. They concluded that the very different color of the rings compared to the moons favored an extrinsic origin, namely formation by tidal breakup of a heliocentric body. However, the comparison of the ring particle surface properties with other Solar System objects should be done again using the same modeling method and more recent data on the satellites (Grundy et al. 1999; Buratti et al. 2002; Cruikshank et al. 2003) which cover a significant wavelength coverage (typically from UV to $2.5 \mu \mathrm{m}$ ).

- The spatial resolution of the observations was sufficient to show a difference between the intrinsic compositions of rings: impurities in the $\mathrm{C}$ ring particle frost must be distributed within the structure of the icy grains or be completely covered by the frost, while the dark matter is intimately mixed in the case of the A and B rings. The dark matter can be included in an icy regolith both by radiation darkening of the organics and meteoritic bombardment. More precisely, the peculiar composition of $\mathrm{C}$ ring frost suggests a model in which a part of the endogenic, intramixed organic red material was destroyed by UV and other radiation, creating intra-mixed C-rich material and providing a flat darkening agent. As an alternate to radiation, once carbon gets to be a significant constituent, it is subject to impact pulverization and mixing within ice grains.

The amorphous carbon impurities mixed intimately with water ice in the A and B rings could come from meteoritic bombardment, as proposed by Cuzzi \& Estrada (1998). A discussion about this dark material is presented in Poulet \& Cuzzi (2002). It is well known that darkening due to meteoroid material that becomes mixed with the ring material appears to give ages much shorter than the Solar System (Doyle et al. 1989; Cuzzi \& Estrada 1998). However, uncertainty in the meteoroid flux could allow the rings to be as old as the Solar System (Dones 1991; Cuzzi \& Estrada 1998).

- Two non-water-ice features were found in the $\mathrm{C}$ ring spectrum, which may be due to some amount of $\mathrm{C}-\mathrm{H}$ on the $\mathrm{C}$ ring particles. However, further laboratory and modeling analysis as well as higher $S N R$ observations in the 3$5 \mu \mathrm{m}$ are required to confirm the features and to identify the non-icy components. The Cassini orbiter now on its way to Saturn is expected to provide clear answers.

\section{References}

Benedix, G. K., Roush, T. L., Owen, T. C., et al. 1998, BAAS, 30, 4409

Brown, M. E. 2000, AJ, 119, 977

Brown, M. E., \& Calvin, W. M. 2000, Science, 287, 107

Buie, M. W., \& Grundy, M. 2000, Icarus, 148, 324

Buratti, B. J., Hicks, M. D., Tryka, K, A., Sittig, M, S., \& Newburn, R. L. 2002, Icarus, 155,375

Clark, R. N. 1980, Icarus, 44, 388

Clark, R. N., \& McCord, T. B. 1980, Icarus, 43, 161

Clark, R. N., Swayze, G. A., Gallagher, A. J., King, T. V. V., \& Calvin, W. M. 1993, U.S. Geological Survey Open File Report 93-592, 1340 pages

Cruikshank, D. P., Roush, T. L., Bartholomew, M. J., et al. 1998, Icarus, 135, 389

Cruikshank, D. P., Roush, T. L., Owen, T. C., et al. 1999, BAAS, 31, 1073

Cruikshank, D. P., Owen, T. C., Dalle Ore, et al. 2003, Icarus, submitted

Cuzzi, J. N., \& Estrada, P. R. 1998, Icarus, 132, 1 
Cuzzi, J. N., Lissauer, J. J., Esposito, L. W., et al. 1984, Saturn's rings, Properties and processes; in Planetary rings, ed. R. Greenberg, \& A. Brahic (University of Arizona Press), 73

Cuzzi, J. N., French, R. G., \& Dones, L. 2002, Icarus, 158, 199

Dalle Ore, C. M., Cruikshank, D. P., Owen, T. C., et al. 1999, BAAS, 310103

Doyle, L. R., Dones, L., \& Cuzzi, J. N. 1989, Icarus, 80, 104

Dones, L. 1991, Icarus, 92, 194

Epstein, E. E., Janssen, M. A., \& Cuzzi, J. N. 1984, Icarus, 58, 403

Esposito, L. W., Cuzzi, J. N., Holberg, J. B., et al. 1984, Saturn's rings - Structure, dynamics, and particle properties, in Saturn, ed. T. Gehrels, \& M.S. Matthews (University of Arizona Press), 463

Estrada, P. R. \& Cuzzi, J. N. 1996, Icarus, 122, 251

Grundy, W., \& Schmitt, B. 1998, J. Geophys. Res., 103, 25, 809

Grundy, W., Buie, M. W., Stansberry, J. A., Spencer, J. R., \& Schmitt, B. 1999, Icarus, 142, 536

Hapke, B. 1981, J. Geophys. Res., 86, 3039

Hudgins, D. M., Sandford, S. A., Allamandola, L. J., \& Tielens, A. G. 1993, ApJS, 86, 713

Jenniskens, P., \& Blake, D. F. 1996, ApJ, 473, 1104

Khare, B. N., Sagan, C., Arakawa, E. T., et al. 1984, Icarus, 60, 127

Khare, B. N., Thompson, W. R., Cheng, L., et al. 1993, Icarus, 103, 290

Lebofsky, L. A., Johnson, T. V., \& McCord, T. B. 1970, Icarus, 13, 226

Lebofsky, L. A., \& Fegley, M. R. Jr. 1976, Icarus, 28, 379
McCord, T. B., Hansen, G. B., Matson, D. L., et al. 1999, JGR, 104, 11827

McDonald, G. D., Thompson, W. R., Heinrich, M., Khare, B. N., \& Sagan, C. 1994, Icarus, 108, 137

Moroz, L.V., Arnold, G., Korochantsev, A. V., \& Wasch, R. 1998, Icarus, 134, 253

Owen, T. C., Cruikshank, D. P., Dalle Ore, C. M., et al. 2001, Icarus, 149,160

Poulet, F., Karkoschka, E., \& Sicardy, B. 1999, J. Geophys. Res., 104, 24,095

Poulet, F., \& Cuzzi, J. N. 2002, Icarus, 160, 350

Poulet, F., Cuzzi, J. N, French, R. G., \& Dones, L. 2002a, Icarus, 158, 224

Poulet, F., Cuzzi, J. N, Cruikshank, D. P., Roush, T., \& Dalle Ore, C. M. 2002b, Icarus, 160, 313

Rayner, J. T., Toomey, P. M., Onaka, A. J., et al. 2003, PASP, 115, 362

Rouleau, F., \& Martin, P.G. 1991, ApJ, 377, 526

Roush, T. L., Pollack, J. B., Witteborn, F. C., \& 1990, Icarus, 86, 355

Roush, T. L., Cruikshank, D. P., Owen, T. C., et al. 1998, BAAS, 30, 1100

Shkuratov, Y., Starukhina, L., Hoffmann, H., \& Arnold, G. 1999, Icarus, 137, 235

Smith, B. A., Soderblom, L., Beebe, R. F., \& 1981, Science, 212, 163

Vacca, W. D., Cushing, M. C., \& Rayner J. T. 2003, PASP, 115, 389

Warren, S. G. 1984, Appl. Opt., 23, 1206 